aus diesem Grund leichter Harnsäure ausfallen lässt, respective ans Harnsäurefilter abgiebt. 2) Die Gicht befällt weit häufiger Männer als Frauen; man sieht z. B. nicht selten in erblich disponirten Familien sämmtliche Brüder an Gicht erkrankeu, sämmtliche Schwestern gesund bleiben. Schwerlich lässt sich in Lebensweise und Functionen beider Geschlechter ein anderer plausibler Grund für diese Verschiedenheit auffinden, als das verschiedene Verhalten im Genuss von Spirituosen. 3) Bei vielen Gichtikern, welche zur. Zeit die Harnsäure "gut gelöst" haben, kann man solche durch vermehrte Alkoholzufuhr "schwer löslich" machen; bei manchen sehr disponirten Männern kann man durch vermehrte Alkoholzufuhr Gichtanfälle prompt hervorrufen; endlich kann man keinen Gichtiker heilen ohne Regulirung seiner Alkoholzufuhr.

Es wäre meines Erachtens leicht verständlich, wenn chronischer Alkoholgenuss die Nieren einzelner Menschen derart beschädigte, dass die Ausscheidung der am schwersten löslichen Stickstoffsubstanz Noth leidet; ja es fehlt nicht einmal ein pathologischanatomischer Befund, welcher vielleicht hierher zu beziehen ist. Bei der Gichtniere findet man nämlich neben Uratablagerungen in der Substanz, nach Vir chow (Berl. klin. Wochenschr. 1884, No. 1) an der Oberfläche der Nieren Spuren multipler Entzündung, eine Art kleiner Narben. Virchow schreibt dieselben der schädlichen Wirkung einer zu concentrirten Harnsäurelösung auf das von ihr passirte Nierengewebe $\mathbf{z u}$; da aber Uratablagerungen an diesen Stellen gerade fehlen, so könnte man diese partiellen Schrumpfungen vielleicht auch der Wirkung des Alkohols zuschreiben. Ein Urtheil hierüber steht mir allerdings nicht zu. Neben der schädlichen Wirkung auf die Nieren kann man auch an eine Beschädigung der Leber, der Bildungsstätte der Harnsäure, und an Störung der (Magen-) Verdauung denken, deren Einfluss auf die Beschaffenheit des Urins bekannt ist.

Man trifft viele Gichtkranke, welche Spirituosen niemals im Uebermaass genossen haben, es scheint vielmehr bei Disponirten eine verhältnissmässig kleine Zufuhrmenge zur Hervorrufung der Krankheit zu genügen. Gichtiker, welche gar nie oder nur ganz selten und dann ganz kleine Mengen Spirituosen consumiren, erinnere ich mich nicht gesehen zu haben. Die (vielfach ererbte) Disposition, welche zur Entstehung der Gicht nothwendig ist, ist ihrem Wesen nach gerade so unbekannt, wie z. B. die vorhandene oder mangelnde Disposition einzelner Menschen oder einzelner Thierclassen bestimmten Infectionskrankheiten gegenüber. Bei erblich Disponirten ist harnsaure Diathese schon im Jugendalter zu erkennen, so beobachtete ich Spuren derselben bei einem 5 jährigen Knaben, in dessen Mannesstamm die Gicht schon seit 200 Jahren erblich sein soll, und dessen Vater schwer gichtkrank war, als er in meine Behandlung kam; deutlich ausgesprochen fand ich harnsaure Diathese bei einem 15jährigen und 17 jährigen Kuaben, deren Vater und Grossvater an Gicht litten.

Dass Gicht ausschliesslich durch Alkoholbeschädigung herbeigeführt werde, will ich nicht behaupten; warum sollte es nicht auch andere chronisch einwirkende Schädlichleiten geben, welclse die Harnsäureausscheidung hindern? Ich hatte in einigen Fällen den Eindruck, dass chronische Störung der Magenverdauung zu Gicht geführt habe. Da nun aber Magenaffectionen eine sehr häufige Folge der Gicht sind, ist im einzelnen Falle schwer darüber zu entscheiden, was das ursprüngliche ist.

Der gewöhnliche Verlauf der Gicht stimmt vollkommen mit der

\section{Zur Lehre von der Harnsäure und Gicht.} Von Dr. W. Camerer, Urach (Württemberg).

(Schluss aus No. 10.)

Es kanu nun keinem Zweifel unterliegen, dass bei weitem in den meisten Fällen die Gicht durch chronische Einwirkung des Alkohols entsteht. Garrod spricht sich auf Grund seiner reichen Erfahrung geradezu dahin aus, dass es ohne Genuss von Spirituosen keine Gicht gäbe, was vielleicht $z u$ viel gesagt ist. - Zur Begrïndung der Ansicht, dass die Gicht (bei Disponirten) eine Wirkung des Alkohols sei, lassen sich folgende, nach meiner Ansicht durchschlageude Gründe anführen: 1) Nach Pfeiffer ist im Urin der meisten Männer die Harnsäure "mangelhaft gelöst, " im Urin von Frauen, Kindern und Greisen "gut gelöst"; d. h. der Urin der Männer zeigt, wenn auch nicht zu voller Stärke entwickelt, so doch mehr oder weniger angedeutet die charakteristische Eigenschaft des Gichturins. Ich habe auf Grund meiner Erfahrungen denselben Eindruck von der Sachlage bekommen, wie Pfeiffer; erinnere übrigens daran, dass der Urin von Männern relativ harnsäurereicher ist, als der von Kindern und Frauen und vielleicht nur

1) P. Guttmann, Real-Encyklopädie der gesammten Heilkunde II. Aufl. Band I p. 580 . Anschauung, dass es sich bei derselbell um lang fortgesetzte, im Tagesmittel minimale Harnsäureretention handle. Meist erst im mittlern Mannesalter, nachdem die Alkoholeinwirkung schon viele Jahre, die Harnsäureretention wohl auch schon geraume Zeit gedauert hat, kommt es zum ersten, kurz dauernden Anfall, erst nach einer Anzahl von weiteren Jahren dauern die Anfälle statt einiger Tage wochenlang, endlich kommt es nicht mehr zu heftigen Anfällen, aber die Kranken werden gar nie ganz frei von Beschwerden. Neben diesem Ablauf der Krankheitsanfälle beobachtet man eine immer zunehmende Ablagerung von Harnsäure im Körper in Gestalt sogenannter Tophi. Die stärkere Beschädigung der Niere, welche nach langem Bestehen der Gicht häufig eintritt, macht die klinischen Symptome der partiellen Schrumpfniere. - Die zahlreichen Erklärungsversuche, warum die Gichtentzündung und die Tophi mit Vorliebe besondere Gewebe und besondere Körperstellen befällt, scheinen mir nicht befriedigend, ohne dass ich etwas besseres an ihre Stelle zu setzen wïsste. Der acute Gichtanfall entsteht, wenn die Safte mit Harnsäure beladen sind, durch mannichfache äussere Anlässe, in vielen Fällen auch ohne nachweisbare specielle Ursache. Eine solche wird auch nicht immer nöthig sein, die Ueberladung des Körpers mit Harnsäure kann doch nicht ohne Grenzen fortschreiten, schliesslich muss eine Gesundheitsstörung eintreten. Als äussere Anlässe kann ich erwähnen: vorübergehende reichliche Harnsäureerzeugung, sei es durch starke Eiweisszufuhr, sei es durch 
Fieber, ${ }^{1}$ ) bei welchem grosse Mengen Körpereiweiss zersetzt werden; ungewöbnlich reichliche Alkoholzufuhr; Verdauungsstörungen; anstrengende Märsche bei grosser Hitze, welche zu einer Eindickung des Blutes und Urins führen. Der günstige Einfluss des Ueberstehens acuter Anfälle auf das Gesammtbefinden beruht vielleicht auf relativ vermehrter Harnsäureausscheidung während des Anfalls, also Verminderung des im Körper aufgespeicherten Stoffes. In ausnahmsweise schweren Fällen schon im Beginn der Gicht, gewöhnlich aber erst nach langjährigem Bestehen, genügt nicht ein Anfall, sondern es folgen sich acute Anfälle in zahlreichen Gelenken viele Wochen lang, bis Genesung eintritt, hier wird eben die Menge der aufgespeicherten Harnsäure erheblich grösser sein. Bei Gichtkachexie ist die Eiweisszufuhr, entsprechend den schlechten Ernährungsverhältnissen, minimal, entsprechend gering auch die tägliche Eiweisszersetzung und Harnsäurebildung, und so mag hier der Gehalt des Blutes an Harnsäure wieder klein werden, daher die acuten Anfälle ausbleiben, wogegen zahlreiche sich nicht mehr zurückbildende Ueberbleibsel von früheren Anfällen im Körper vorhanden sind; es handelt sich hier offenbar weniger um neue Beschädigungen, als um einen Zustand der Invalidität. Blutanalysen in verschiedenen Stadien der Krankheit werden über die Berechtigung dieser Anschauungen Aufschluss geben. Die Bildung der Tophi wirkt insofern günstig, als dabei aufgespeicherte Harnsäure in unlöslicher Form abgelagert und der Circulation entzogen wird (denn das Gewebe der von den Tophi befallenen Stellen ist nekrotisch), und es mag die heilsame Einwirkung des Anfalls zum Theil auch hierauf beruhen.

\section{Diagnose der Gicht.}

So leicht dieselbe bei dem gewöhnlichen Verlaufe der Krankheit ist, so schwierig in einzelnen Fällen mit abnormem Verlauf. Schon dann, wenn die Gicht zwar acut beginnt, aber wenn von Anfang an grosse Gelenke befallen werden, und wenn statt eines kurzdauernden Anfalles eine Reihe solcher im Laufe mehrerer Wochen erfolgt, ist Verwechslung mit acutem Gelenkrheumatismus möglich und namhaften Aerzten vorgekommen, vollends aber ist Verwechslung mit "chronischem Gelenkrheumatismus" möglich, wenn die Gicht von Anfang an in chronischer Form auftritt. Der sogenannte chronische Gelenkrheumatismus (auch Arthritis deformans, polymorpha u. s. w. genannt) ist eine zwar häufig vorkommende, aber nicht genügend bekannte Krankheit, daher seien einige Bemerkungen über denselben eingeschaltet. Er tritt in zwei allerdings nicht scharf geschiedenen Formen auf, von welchen die erstere häufiger jüngere Leute etwa bis zum 50. Lebensjahr, die zweite häufiger ältere Leute befällt.

Das Krankheitsbild der ersten Form schliesst sich zuweilen unmittelbar an das des subacuten Gelenkrheumatismus an, oft ist der Verlauf viel schleppender, indem die Krankheit ohne oder höchstens mit vorübergehendem geringem Fieber jahrelang sich hinzieht. Es giebt zwischen diesen ganz chronisch verlaufenden Fällen und den stürmisch verlaufenden Fällen des acuten Gelenkrheumatismus alle denkbaren $\mathrm{Zwischenstufen.} \mathrm{Die} \mathrm{grossen} \mathrm{Gelenke} \mathrm{werden} \mathrm{beim} \mathrm{chro-}$ nischen Rheumatismus so gut befallen wie die kleinen; es wechselt Verschlimmerung und Besserung, zuweilen tritt dauernde oder nur vorübergehende Genesung ein.

Die Gelenke sind manchmal durch erhebliche Ergüsse in die Gelenkhöhle ausgedehot, in anderen Fällen kommt die Anschwellung lediglich auf Rechnung der, die Gelenkkapsel umgebenden Weichtheile. Nicht selten nimmt zwar die Entzündung ab, aber das Gelenk wird halbsteif, knarrt bei Bewegungen, wird deform, kurz es tritt der Zustand ein, welchen man als Arthritis deformans oder sicca bezeichnet, und welcher der Ausgang auch anderer Gelenkkrankheiten zu sein scheint.

Die zweite Form befällt häufiger die kleinen Gelenke, namentlich der Finger. Die Gelenke werden plump, ab und zu schmerzhaft und bekommen kleive Höcker, welche von den Tophi bei (harnsaurer) Gicht oft nicht zu unterscheiden sind; ein bezeichnender Name für diese Krankheit wäre Arthritis nodosa. Zuweilen werden Hand und Finger verkrümmt und steif, wie man hier zu Lande sagt „ganz contract." Von den Laien wird diese Krankheit immer als Gicht bezeichnet und hat in der That nit manchen Fällen chronischer Gicht die grösste Aehnlichkeit. Auch diese zweite Form scheint mit dem acuten Gelenkrheumatismus in einem gewissen $\mathrm{Zu}$ sammenhange zu stehen. Personen, welche schon öfters an letzterem gelitten haben, erkranken manchmal an solch chronischer Entzündung kleiner Gelenke, so zu sagen als Ersatz für ausbleibende Anfälle der schwereren Krankheit.

Ich habe in zahlreichen Fällen gefunden, dass Mitglieder gewisser Familien überhaupt häıfiger als gewöhnlich von Gelenkrheumatismus befallen werden, und zwar zeigen einzelne Glieder

J) Ich habe theils erlebt, theils von Kranken gehört, dass sich Gichtanfälle unmittelbar an eitrige Anginen, an Pneumonieen, an mit Fieber verbundene Körperver]etzungen anschlossen. mehr Vorliebe für die acuten, andere mehr für die chronischen Formen; wieder andere wechseln $z$ wischen beiden ab.

Der acute Gelenkrheumatismus gilt zur Zeit für eine durch Coccen: verursachte Infectionskrankheit; ich glaube, man hat Grund, auch für den chronischen Gelenkrheumatismus einen ähnlichen Ursprung anzunehmen. Ausser den bisher angeführten Thatsachen, welche einen nahen Zusammenhang beider Krankheiten wahrscheinlich machen, spricht bein chronischen Rheumatismus folgendes für diese Annahme: Da die Krankheit bald dieses, bald jenes Gelenk befällt, handelt es sich um eine im Körper transportable Ursache; ferner lassen sich alle anderen chronisch einwirkenden Schädlichkeiten, chemische, mechanische, wenn jüngere Personen befallen werden, auch Altersveränderungen ausschliessen: endlich kommt es auch beim chronischen Gelenkrheumatismus nicht ganz selten zu Herzklappenfehlern, welche ja meist von Coccen verursacht sind.

Die Differentialdiagnose zwischen chronischer Gicht und chronischem Gelenkrheumatismus, namentlich der Arthritis nodosa wird folgende Punkte zu berücksiclitigen haben: 1) Eine sorgfältige Aufnahme der Kranken- und Familiengeschichte. 2) Nachweis von Harnsäureansammlung im Körper, nach Garrod im Blut oder der Blasenpflasterflüssigkeit; für den praktischen Arzt aber viel bequemer durch Nachweis deutlicher harnsaurer Tophi, welche häufig im $\mathrm{Ohr}$ knorpel von Gichtiker'n vorhanden sind; die Tophi an andereu Körperstellen sind allerdings nicht so leicht als harnsaure Ablagerungen zu erkennen. Zuweilen entleert ein oberflächlich gelegener Tophus mit oder ohne Eiterung kreideartige Massen, welche unter dem Mikroskop sich als Nadeln vou saurem harnsaurem Natron erweisen. 3) Nachweis der abnorm leichten Ausfällbarkeit der Harnsäure aus dem Urin. Die Methode von Pfeiffer vermittels des Harnsäurefilters erfordert den Besitz einer chemischen Waage und dürfte für die meisten praktischen Aerzte zu umständlich sein; sie leistet, glaube ich, nicht mehr, als die unmittelbare Beobachtung des Urins. Ich verfahre dabei folgendermaassen: Die 24 stündige Urinmenge der zu untersuchenden Person wird durch passende Getränkezufuhr auf das specifische Gewicht 1010-1020 gebracht es entspricht das bei einem Manne mit gewöhnlicher Ernährung $2500 \mathrm{ccm}$ bis $1500 \mathrm{ccm}$ Urin und einer Flüssigkeitszufuhr von 2 bis $3 \mathrm{l} \mathrm{im} \mathrm{Tag.} \mathrm{Eine} \mathrm{Probe} \mathrm{dieses} \mathrm{Urins} \mathrm{in} \mathrm{einer} \mathrm{Flasche} \mathrm{von}$ $150 \mathrm{ccm}$ bis $200 \mathrm{ccm}$ Gehalt aufbewahrt wird im Laufe von 24 bis 36 Stunden Krystalle von Harnsäure absetzen, sei es aus dem klaren Urin, sei es aus dem durch Bildung von Uraten trübe gewordenen, wenn harnsaure Diathese vorhanden ist. Zuweilen misslingt der Versuch deshalb, weil der Harnstoff- und Harnsäuregehalt des Urins viel geringer ist, als dem specifischen Gewicht nach zu erwarten wäre; ich habe eininal mehrere Tage hindurch bei einem specifischen Gewicht von 1014 kein Resultat gehabt, der Harnstoffgehalt des Urins wurde bei der Untersuchung zu $1 \%$ gefunden, während ich etwa 1,5\% auf Grundlage meiner Erfahrungen erwartet hatte. Giebt die Untersuchung an $2-3$ Versuchstagen kein Resultat, wo man ein solches erwarten konnte, so kann man den Kranken einige Tage reichlich Wein trinken lassen und alsdann untersuchen. Manchmal muss der Weingeuuss 8-10 Tage fortgesetzt werden, ehe Erfolg eintritt. Dieser Versuch ist aber nicht ganz ungefährlich, er kann bei schweren Kranken einen Anfall provociren; allerdings wird man bei solchen meist ohne Weingenuss zurechtkommen. Eine gewisse Uebung in Beurtheilung des Befundes ist natürlich nothwendig, um richtige Schlüsse zu ziehen. 4) Nach Pfeiffer lässt sich die harnsaure Diathese auch durch subcutane Einspritzung einer Lösung von phosphorsaurem Natron nachweisen; mir fehlt eigene Erfabrung darüber.

Ist man auf die eine oder andere Art darüber sicher geworden, dass der Untersuchte an harnsaurer Diathese leidet, so besteht grosse Wahrscheinlichkeit, aber doch noch keine absolute Gewissheit, dass vorhandene chronische Gelenkentzündungen durch Harnsäurebeschädigung entstanden sind. Es kann zufälliger Weise ein Mensch mit harnsaurer Diathese z. B. an Gichtniere und harnsauren Tophi in den Ohren leiden, nicht aber an Gelenkgicht und gleichzeitig von chronischem Gelenkrheumatismus befallen sein; auch wüsste ich keinen Grund, warum nicht letztere Krankheit einmal zugleich mit Gelenkgicht vorkommen könnte. In solchen zweifelhaften Fällen entscheidet dann der Erfolg der Behandlung, welch letztere bei Gicht von sicherer und rascher Wirkung, bei chronischem Gelenkrheumatismus ziemlich unwirksam ist.

\section{Die Therapie der Gicht.}

Nimmt man an, der acute oder subacute Aufall sei ein Heilungsvorgang, so muss man gegen das Coupiren desselben sein. Solches scheint durch Colchicumpräparate und die Mittel des Dr. Laville (Drastica) in der That bis zu einem gewissen Grad möglich zu sein, aber der häufige Gebrauch der letzteren Mittel beschädigt das Allgemeinbefinden in hohem Maasse. Die meisten Gichtiker, welche viele Anfälle gehabt baben, verzichten schliess- 
lich anf jede Therapie während des Anfalles, nnd es wird wohl am besten sein, wenn man den acuten nud nicht allzn lang sich hinschleppenden subacnten Anfall etwa nuter Anwendung schmerzstillender Mittel, welche für die Gicht selbst indifferent sind, anstoben lässt. Bei denjenigen snbacnten Anfällen dagegen, welche sehr lange Zeit, $1 / 2-3 / 4$ Jahre fortdauern, indem immer wieder nene Räckfälle von Gelenkentzündnngen eintreten, ist ärztliches Eingreifen nothwendig nnd von grossem Erfolge, denn diese endlose Wiederholung von Anfällen rührt meist von nachweisbaren Schädlichkeiten her, welchen sich die genesenden Kranken anssetzen. Ich glanbe, dass nach 1-2 monatlicher Daner weitere Anfälle immer verhütet werden könnten. Die Heilnng erfolgt in solchen Fällen in der Art, dass in den nächsten 2-3 Wochen nach Beginn einer Cnr noch minimale Anfälle eintreten, welche den Kranken kanm belästigen und unr ganz knrze Zeit danern, nach und nach verschwinden anch diese.

Die Behandlnng der Gicht besteht im wesentlichen in Verhiitnng weiterer Harnsänrebeschädignngen, denn die bereits geschehenen können therapentisch nicht mehr rückgängig gemacht werden, wenn sie sich anch von selbst, im Lanfe der Zeit, sehr verbessern. Sie hat folgende Pnnkte zn berücksichtigen:

1. Die Eruährung. In dieser Beziehnng findet man die widersprechendsten Angaben in den Büchern, worans man den Schluss ziehen wird, dass ein ziemlich weiter Spielranm gestattet ist. In der That brancht die Eruährung der Gichtiker keine specifische zn sein, wie etwa bei Znckerkranken, sondern sie richtet sich im wesentlichen nach dem Gesammtbefinden. Ist der Kranke, wie znweilen im Anfang der Gicht der Fall, ein starker Esser, so wird man Veranlassnng haben, gegen das Uebermaass einznschreiten, ist er an Kräften sehr hernntergekommen, so wird man die Eruährung anf alle Weise zn heben suchen. Eine übermässige Tagesproduction an Harnsänre wird man beim Gichtiker natürlich vermeiden müssen, ich lasse an Fleischznfulır abbrechen, wenn die tägliche Harnstoffmenge $30 \mathrm{~g}$ übersteigt (dieselbe dient anch hier als Index für die Harusänremenge). Anch übermässigen Gennss von Essig nnd anderen Sänren wird man selbstverständlich nutersagen. Von grosser Wichtigkeit ist, die Nahrnng so anšnwählen, dass keine Verdanungsstörung entsteht (deun eine solche kann Anlass zn einem Gichtanfalle geben), eine bei Gichtkachexie oft recht schwierige Anfgabe!

2. Die Getränkezufuhr des Gichtikers mnss reichlich sein, damit der Urin nicht allzn concentrirt werde. Getränkemengen von 2-3 1 im Tag entspricht nuter gewöhnlichen Umständen eine Urinmenge von 1500 bis $2500 \mathrm{ccm}$, welche genügt. Grosse Märsche in der Sommerhitze sind von den Kranken, wie schon früher angedentet, zu meiden. Im Fieber ist der Urin concentrirt nnd sehr harnsänrehaltig. Man wird bei fiebernden Gichtikern wohl thun, dnrch besouders reichliche Getränkezufuhr, Unterlassen der Weinbehandlnng, Vorsicht in Znfnhr stickstoff haltiger Nahrungsmittel der Gefahr möglichst vorznbengen. Vielleicht empfiehlt sich der Gebranch von Chinin, welches beim Gesnnden die Harnsänreprodnction beschränken soll. Ob diese Angabe richtig ist, kann ich allerdings nicht ans eigener Erfahrung sagen.

3. Die Verabreichnng kohlensanrer Alkalien ist bei Gicht allgemein üblich, nnd anch ich lasse täglich nud anf die Daner 1 bis $3 \mathrm{~g}$ doppelkohlensanres Natron nehmen. Chloralkalien nnd schwefelsanre Alkalien könuen anf die Reaction des Blntes nnd des Urins nnmöglich einen Einfinss haben, und es ist mir deshalb die Empfehlnng von Chlorlithium oder Kochsalz unverständlich. Kohlensanres Lithion ist in Wasser schwer löslich nnd reizt, in Schn̈ttelmixtnr gegeben, nicht selten den Magen. Die Empfehlnng des Lithions, welche von Garrod ansging, setzt vorans, dass sich die Harnsänre gerade mit dem Lithion im Blnt nnd Urin verbinde, eine nnwahrscheinliche. jedenfalls nicht $\mathrm{zn}$ beweisende Annahme!

Ganz zweckmässig geschieht die Znfnhr der kohlensanren Alkalien in Gestalt eines natürlichen Mineralwassers (Fachinger, Obersalzbrunner etc.), da solche von geeigneter Concentration oder vielmehr Verdünunng and zngleich wohlschmeckend sind. Der hohe Preis dieser Mineralwässer macht aber für viele Kranke den lange fortsgesetzten Gebranch schr nuangenehm oder fast numöglich, und es empfiehlt sich daher, wie von vielen Aerzten geschehen, Herstellung eines künstlichen Mineralwassers, welches im Liter $2-3 \mathrm{~g}$ doppelkohlensaures Natron enthält. Wer Werth daranf legt, kann kleine Mengen kohlensanres Lithion, Chlorlithion, Chlornatrinm etc. beifügen, wie sie in den natürlichen Wassern vorkommen. Für entsprechenden Kohlensänregehalt ist zn sorgen, damit die Mischnng wohlschmeckend ist. Der Gebranch des Wassers geschieht nicht früh morgens, sondern den ganzen Tag über in kleinen Portionen entsprechend der Tagescnrve der Harnsänrebildung.

4. Der wichtigste Pnukt bei der Gichtbehandlnng ist Regnlirung der Alkoholznfnhr. Manche halten Wein nud Bier für schädlicher als kleinere Gaben von Cognac und dergleichen, welche Ansicht ich nicht theilen kann. Sie rïhrt vielleicht daher, dass man den Ge- halt des Weines an Alkohol vielfach nnterschätzt; $100 \mathrm{ccm}$ desselben enthalten im Durchschnitt $10 \mathrm{ccm}$ wasserfreien Alkohols. Am sichersten' wäre es, Gichtikern den Geunss von geistigen Getränken ganz und auf die Daner zu nutersagen. Da aber viele Männer theils ans Gewohnheit nnd wirklichem Bedürfuiss, theils wegen ihrer socialen Stellnng vollständige Abstinenz nicht' durchführen können; handelt es sich darnm, in jedem Einzelfalle das zulässige Maass ansznmitteln. Allgemeine Regeln lassen sich hierfür nicht anfstellen, die Erfahrnng des Ar"ztes muss das Richtige treffen.

5. Nach überstandenem Gichtanfalle ist früh zeitige und energische Uebnng der befallenen Gelenke von grösster Wichtigkeit. Die Gebranchsfähigkeit stellt sich dabei in nnerwartet kurzer Zeit wieder her, anch gelingt es hänfig, Gelenke wieder beweglich zn machen, welche bei früheren Anfällen ans Mangel an Uebung steif geworden sind.

Ich habe nie nöthig gefunden, ansser diesen einfachen Mitteln noch weitere Maassregeln (z. B. warme Bäder) in Anwendnug zn bringen, immer habe ich äberraschende Erfolge anch in verzweifeltell Fällen, welche bisherigen Cnren getrotzt hatten, erzielt. Bei iüngeren Lenten mit verschleppten snbacnten Anfällen genügen 4-8 Wochen, um ein Arzt nud Kranken befriedigendes Resnltat herbeiznführen, bei älteren Lenten mit chronischer Gicht ist voller Erfolg erst im Lanfe von $1 / 1-1 / 2$ Jahr $z$ n erwarten (soweit ein solcher überhanpt möglich ist). - Wenn die Kranken die vorgeschriebene Lebensweise einhalten, so bleiben sie von nenen Anfällen mit Sicherheit frei, anch wenn sie vorher hänfig, z. B. alle Jahre einmal oder halbjährig befallen wurden. Meine Erfahrungen erstrecken sich nnnmehr anf einen Zeitranm von Ј Jahren, nud man darf Kranke, welche so lange frei von Anfällen geblieben sind, während sie vorher lange Zeit hindnrch mehrmals im Jahre acnte Anfälle hatten oder gar nie von Beschwerden frei wnrden, wohl als danernd geheilt bezeichnen.

Nachtrag. Nach Absendnug des Mannscriptes obiger Arbeit hatte ich Gelegenheit, noch einige interessante Untersuchungen über Harnsänre anznstellen, deren Resnltate in Kürze mitgetheilt werden sollen.

\section{Fiebernrine.}

Fall I betrifft einen 10 jährigen Knaben, welcher nach 6 wöchigem Verlanf an acuter Miliartnberculose starb. Die Aftertemperatnren an den 4 Versnchstagen (von welchen einer der letzte Lebenstag war) betrugen $z$ wischen 38,1 nnd 41,1 ; die Untersnchnng fiel in die letzten 4 Wochen der Krankheit. Die Schwanknngen des Befundes an den einzelnen Versuchstagen waren unbedentend, daher ich nur Mittelzahlen für alle 7 Tage gebe, Nahrnugszufuhr fand gar nicht oder nur in sehr beschränktem Maasse statt. Eine Portion Urin, von den letzten 12 Lebensstnnden stammend, wnrde bei der Section ans der Blase gesammelt. - Fall II betrifft eine 32 jährige Fran mit vorgeschrittener schwerer Lnugen- nnd Darmtuberculose. Die Temperaturen an den 6 Versuchstagen (im Lanfe der letzten 3 Lebensmonate), im Mnude gemessen, schwankten zwischen 38,7 nnd 39,7. Die Kranke war änsserst entkräftet, genoss Wein nnd Cognac, aber gar keine oder nur änsserst wenig Nahrnng. - Fall III betrifft einen 38jährigen Mann mit acntem Gelenkrhenmatismus milden Verlanfs in der dritten Woche der Krankheit. Der Kranke ist seit seinem 20. Lebensjahre schon $6 \mathrm{mal}$ vom Rhenmatismns befallen worden, am Versnchstage war die Aftertemperatnr 38,1-38,7, Esslnst gross nnd Nahrnngsznfuhr bedentend. Er verbranchte (seit 3 Wochen schon) im Tag 2 g Natr. salicylic.

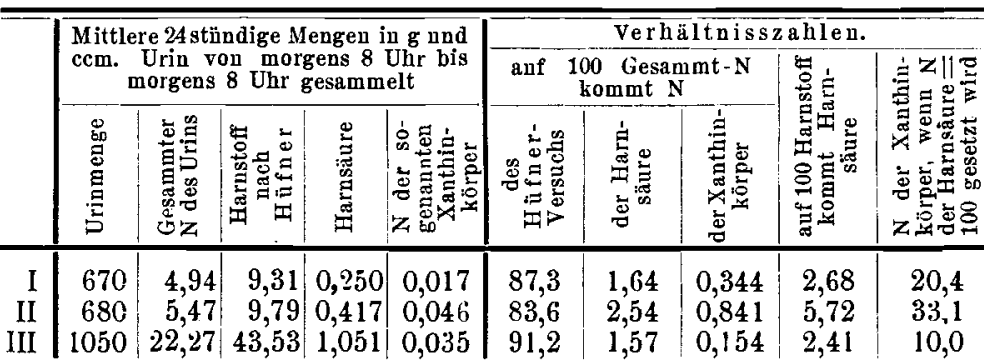

Der Urin von Fall III zeigt bei sehr mässigem Fieber, die bekannten Eigenschaften eines Urins bei reichlicher Eiweissznfubr, nämlich relativ viel Harnstoff, dazn relativ wenig Harnsänre nnd Xanthinkörper, was von dem einige Wochen fortgesetzten Gebranch des Natr. salycil. nnd der Mässignng im Gennss von Wein nnd Bier herrühren könnte. Der Urin in Fall I nnd II zeigt die Eigenschaften eines Urins bei reichlicher Zerstörnng von stickstoff haltigen Körperbestandtheilen, verhältnissmässig wenig Harnstoff, viel Harnsänre nnd viel Xanthinkörper (von welchen nnten die Rede sein wird). Ansserdem sieht man, dass wie in gesunden Tagen, so anch bei schwerem Fieber die relative Harnsänremenge des Kindes elheblich kleiner ist als die des Erwachsenen. 
Relativ kleinen Harnstoffgehalt und relativ grosse Harnsäuremenge hat auch der während der Verdauung entstandene Urin, relativ kleinen Harnstoffgehalt und normale Harnsäuremenge hat, soviel bisher bekannt, der Urin des Gesunden bei ungewöhnlich geringer Eiweisszufuhr; die relative Menge der Xanthinkörper scheint in beiden letzteren Fällen (welche übrigens noch nicht genügend in dieser Richtung untersucht sind) die normale zu sein.

\section{Urin von Männern und Frauen.}

Ich sammelte an 2 Versuchstagen den 24 stündigen Urin von 5 Ehepaaren gebildeten Standes, ohne allen Verlust, die Männer im Alter von 33-60, die Frauen von 25-49 Jahren. Eine der Frauen säugte. Die Mahlzeiten der einzelnen Ehepaare waren natürlich gemeinsam, womit aber nicht gesagt ist, dass die Auswahl unter den vorhandenen Speisen von beiden Betheiligten ganz gleichmässig geschah. Versuchszeit war Mitte Juni 1890. Die Männer genossen jedenfalls mehr Wein und Bier als die Frauen, welch letztere sich dieser Getränke nicht ganz enthielten. Das Ergebniss war folgendes:

\begin{tabular}{|c|c|c|c|c|c|c|c|c|c|c|}
\hline & \multirow{2}{*}{\multicolumn{2}{|c|}{$\begin{array}{l}\text { Mittlere } 2 \\
\text { gr und c }\end{array}$}} & \multirow{2}{*}{\multicolumn{3}{|c|}{$\begin{array}{l}24 \text { stüudige Mengen in } \\
\text { ccm, auf eine Person } \\
\text { berechnet }\end{array}$}} & \multicolumn{5}{|c|}{ Verhaltnisszahle } \\
\hline & & & & & & \multicolumn{3}{|c|}{$\begin{array}{l}\text { auf } 100 \text { Gesammt-N } \\
\text { des Urins kommt } N\end{array}$} & \multirow{2}{*}{ 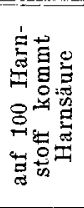 } & \multirow{2}{*}{ 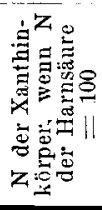 } \\
\hline & 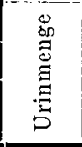 & 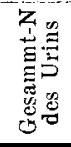 & 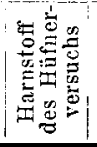 & 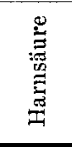 & 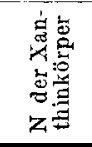 & 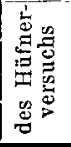 & 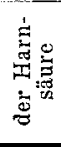 & 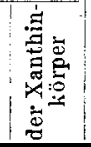 & & \\
\hline & 94 & 14,8 & 29,00 & & 02 & 91,2 & 1,70 & 0,182 & & \\
\hline Frau & 1499 & 11,89 & 22,93 & 0,552 & 0,0360 & 91,2 & 1,55 & 0,303 & 2,41 & 19,6 \\
\hline
\end{tabular}

Nach der Tabelle ist bei den Frauen die relative Harnsäuremenge kleiner, die relative Menge des Xanthin- $\mathrm{N}$ grösser als bei den Männern. Die Differenzen sind allerdings derart, dass sie beinahe in den Grenzen der analy tischen Versuchsfehler $( \pm 1 \%$ ) liegen, doch ist sehr unwahrscheinlich, dass bei den 4 in Betracht kommenden Analysen (2 für Männer, 2 für Frauen) jedesmal die grösstmöglichen Fehler vorgekommen und jedesmal in entgegengesetztem Sinne ausgefallen sind. Der Befund findet wohl seine Erklärung durch folgenden Abschnitt:

\section{Ursache der Schwankungen im relativen Harnsäure-} gehalt gesunder Menschen.

Dieselben sind bei ziemlich einförmiger Kost auffallend gross, so fand ich bei mir selbst im Winter $1889 / 1890$ an 5 Versuchstagen auf 100 Harnstoff 2,5-3,2 Harnsäure, und es entsprach im allgemeinen einem grossen Harnsäuregehalt ein kleiner Gehalt an N der Xanthinkörper und umgekehrt. Auffallend kleine relative Harnsäuremengen und grosse Mengen von Xanthin- $\mathrm{N}$ fand ich zu meiner Ueberraschung im Laufe dieses Sommers bei einigen Gichtkranken; nämlich 100 Harnstoff: 2,3-2,7 Harnsäure und auf 100 Harnsäure N 15-26 Xanthin-N; bei einer Dame, welche an ausgesprochner harnsaurer Diathese, schlechtem Magen, allerlei nervösen Symptomen, namentlich nervösem Herzklopfen litt - diese Erscheinungen, nicht aber Gelenkgicht sind bei harnsaurer Diathese der Damen gewöhnlich - fand ich gar 100: 1,7-2,0; und auf 100 Harnsäure-N 19 bis 38 Xanthin-N. Bei 3 Versuchen an mir selbst, welche ich demzufolge anstellte, fand ich 100 Harnstoff: $2,0: 2,3: 2,4$ Harnsäure; auf $100 \mathrm{~N}$ der Harnsäure kam 25;30;23 Xanthin-N. Die Ursache hiervon liegt ohne Zweifel darin, dass der Sommer eine Veränderung der Kost mit sich bringt: Die genannten Kranken verzehrten, wie ich selbst, während der Versuchszeit viel Salat, grüne Gemüse, Kirschen und Erdbeeren, welche eben reif waren. - Ueber die sogenannten Xanthinkörper sti folgendes bemerkt: Versetzt man einen ammoniakalisch gemachten Urin mit Lösung von Silbernitrat, so entsteht ein Niederschlag, welcher alle Harnsäure und eine Anzahl andrer N-haltiger Urinbestandtheile enthält, welch letztere nur theilweise bekannt sind und unter dem Namen „Xanthinkörper" zusammengefasst werden. Dass die Xanthinkörper des Fieberurins grösstentheils andere sind, als die Xanthinkörper des Sommerurins, ist unzweifelhaft. Bei dem Urin der Fieberkranken sowohl, als bei den Sommerurinen findet man eine Vermehrung des Xanthin-N im Verhältniss zum Harnsäure- $\mathrm{N}$, bei dem ersten gleichzeitig eine Vermehrung des Harnsäure-N im Verhältniss zum Gesammt-N, d. h. gl eich zeitig wächst im Fieber die Ausscheidung der Harnsäure und der Xanthinkörper, nur letztere in stärkerem Maasse als erstere. Bei den Sommerurinen wächst zwar die Ausscheidung des Xanthin-N, aber die Harnsäureausscheidung nimmt etwa in demselben Verhältniss ab. Ueber weitere Versuche, welche die Erscheinung vollständig aufklären, wird in einer demnächst erscheinenden Arbeit in der ,Zeitschrift für Biologie" $^{\text {"6 }}$ berichtet werden. 